ROSA JORDÁ BORREL*

\title{
CONTRIBUCIÓN ANDALUZA A LA FORMACIÓN DE UN ESPACIO TRANSRONTERIZO EN LA RONTERA HISPANO-MARROQUÍ
}

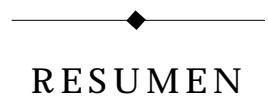

En este artículo nos vamos a centrar en la manera en que está evolucionado el flujo del comercio exterior entre Andalucía y Marruecos, y cuáles han sido las estrategias de los agentes económicos andaluces para penetrar en el mercado marroquí aprovechando la expansión de la demanda y los bajos precios de la mano de obra, todo ello teniendo en cuenta que la zona de librecambio se inició en 1996 y todavía no ha pasado tiempo suficiente para hacer un análisis profundo de la cuestión. En segundo lugar, queremos medir el nivel de participación de la Comunidad Autónoma Andaluza en la construcción de infraestructura (puertos, centrales eléctricas, térmicas, aeropuertos, telecomunicaciones, sanidad) y equipamiento para colectividades locales, regionales y nacionales marroquíes, puesto que constituye otra forma de penetración en el mercado alauita, y puede considerarse también una manera de conocer la participación de Andalucía en el proceso de desarrollo de la zona de libre comercio marroquí, ya que está inserta doblemente en dicho proceso, como región de la Unión Europea (UE) y por ser puerta de Europa.

\section{A BSTRACT}

This article will be centered in the way of evolving flow of the exterior trade between Andalusia and Morocco, and the strategies of the economic Andalusian agents to penetrate within the Morocco market taking advantage of the growing demand and the low prices of labor, all of these without forgetting that the free exchange zone started in 1996, and is too early to make a deep analysis of this matter. Secondly, we want to measure the level of participation of the Andalusian Autonomous Community to build infrastructure (ports, electric stations, thermoelectric, airports, telecommunications, health) and equipping local, regional and national Moroccan communities, due to it constitutes another way to penetrate in the Andalusian market, and can be considered another way of Andalusia sharing in the development process of the Moroccan free trade zone, since it is twice inserted in said process as a region of the European Union and as a door of Europe.

* Profesora investigadora de la Universidad de Sevilla. Correo electrónico: rjborrell@terra.es y borrell@us.es 


\section{INTRODUCCIÓN}

Las relaciones entre Andalucía y Marruecos han estado marcadas por la tradición histórica y los vínculos culturales derivados de un pasado en común, pero casi nunca se han apoyado en razones económicas y políticas. Incluso más recientemente, cuando seempezó a difundir en España la idea de la cooperación institucional, la Junta de Andalucía comenzó a practicar ésta en Marruecos (1988) a partir de iniciativas culturales y de asistencia humanitaria, pues, en el ámbito de las relaciones económicas y políticas, ha existido casi siempre un desentendimiento.

En el terreno económico ha persistido, a través del tiempo, un choque de intereses en torno a los recursos naturales situados en la plataforma continental y en el Sahara occidental (minas de fosfatos), e incluso por la competencia entre ambos países en lo que a la exportación de productos agrarios mediterráneos se refiere. Como Andalucía y Marruecos (norte) presentan características geomorfológicas y climáticas semejantes, las actividades principales de estos países son la exportación de productos agrarios y la pesca, las cuales representan $30 \%$ y $55 \%$ del total de sus ingresos respectivamente. N o obstante, es importante resaltar que la renta per capita marroquí equivale al $16 \%$ de la andaluza, 50\% de las carreteras marroquíes no están asfaltadas, únicamente $20 \%$ de la población rural de Marruecos tiene abastecimiento de agua potable, y sólo $15 \%$ de dicha población dispone de electricidad en sus hogares. Por otra parte, si bien es cierto que existen grandes contrates entre un lado y otro de la frontera, como el nivel de industrialización e internacionalización, el cual en el lado andaluz es todavía bajo, los conflictos pesqueros y agrícolas siguen teniendo una repercusión importante en la economía de esta última, de ahí que ambos espacios hayan sido rivales en el terreno económico, y esa problemática haya influido en las relaciones hispano-marroquíes, dado que las cuestiones pesqueras y agrarias trascienden en el lado español más allá de Andalucía.

En el ámbito político, el desentendimiento se ha debido a las características geoestratégicas del estrecho de Gibraltar, que han Ilevado a Marruecos y a España al recelo, e incluso a tensiones no 
del todo superadas, hoy explicadas fundamentalmente por la existencia de Ceuta y Melilla como ciudades españolas.

Pero en la actualidad han surgido nuevos elementos que están redefiniendo las relaciones políticas y económicas hispano-marroquíes. N os referimos a las buenas relaciones que mantienen España y Marruecos a nivel de Estado, y a los cambios derivados de la entrada de nuestro país a la Unión Europea (UE), especialmente aquellos que tienen que ver con la aplicación de la política euromediterránea. Aunque el menor peso político de España frente a otras potencias europeas también ha contribuido a suavizar las tensiones políticas recientes.

La política mediterránea de la UE ha iniciado una nueva fase de cooperación "euromediterránea" a través de las Cumbres Económicas y Sociales de Barcelona en 1995, de Malta en 1997, de Casablanca en 1998, lo que ha permitido que se dé una corresponsabilidad entre los países del norte, estey sur dela cuenca, conllevando, al mismo tiempo, una idea de reciprocidad y de simetría, idea que se aplica a un espacio profundamente asimétrico en términos de niveles de desarrollo, de situación demográfica, de posicionamiento en la economía mundial y de impactos del nuevo régimen comercial (muy débil al norte y consi derableen el sur). Deahí queesta distorsión entresimetría querida y asimetría efectiva puede constituir un desafío para dinamizar el desarrollo de los países del sur y del este del Mediterráneo, pero ella constituye también un riesgo serio de fracaso, no sólo en el norte de África, sino también en las regiones periféricas europeas del Mediterráneo, como lo sería el caso de Andalucía. La conciencia de este riesgo en el Mediterráneo sur ha llevado a Bruselas a poner en marcha acciones preventivas (acciones MEDA) y correctoras.

En este contexto, el acuerdo deasociación entrela UE y Marruecos ha dado lugar a la apertura gradual de una zona de libre comercio a partir de 1996 y durante un periodo de 12 años, haciendo distinción entre el trato concedido a los productos industriales por una parte, y a los agrícolas y pesqueros, por la otra. Así, Marruecos está desmantelando progresivamente los niveles arancelarios y no arancelarios del sector industrial, mientras queel mercado europeo ya está abierto a la producción marroquí. 
En el caso de los productos agrarios y pesqueros, Bruselas los ha utilizado como moneda de cambio para solucionar los problemas conflictivos dela UE con el Magreb (desequilibrio demográfico nortesur, desempleo, el tema del agua, de la energía, del medio ambiente, etcétera), obligando a sus regiones periféricas a internacionalizarse, a adoptar un posicionamiento en la economía mundial, y a pasar rápidamente a la economía de la información sin haberse preparado lo suficiente.

En este artículo nos vamos a centrar en la manera en que está evolucionado el flujo del comercio exterior entre Andalucía y Marruecos, y cuáles han sido las estrategias de los agentes económicos andaluces para penetrar en el mercado marroquí aprovechando la expansión de la demanda y los bajos precios de la mano de obra, todo ello teniendo en cuenta que la zona de librecambio se inició en 1996 y todavía no ha pasado tiempo suficiente para hacer un análisis profundo de la cuestión. En segundo lugar, queremos medir el nivel de participación de la Comunidad Autónoma Andaluza en la construcción de infraestructura (puertos, centrales eléctricas, térmicas, aeropuertos, telecomunicaciones, sanidad) y equipamiento para colectividades locales, regionales y nacionales marroquíes, puesto que constituye otra forma de penetración en el mercado alauita, y puede considerarse también una manera de conocer la participación de Andalucía en el proceso de desarrollo dela zona de libre comercio marroquí, ya que está inserta doblemente en dicho proceso, como región de la UE y por ser puerta de Europa.

\section{FLUJO DE LAS IMPORTACIONES}

Para analizar el primer punto hemos estudiado los intercambios comerciales (exportación e importación) A ndalucía-Marruecos, diferenciando entre la evolución del flujo de productos y el análisis de las empresas participantes. En el primer caso disponíamos de la serie de datos correspondiente a 1989-2000, habiéndola tratado con medias móviles de tres años en tres años, a fin de corregir la inestabilidad del flujo. Para el estudio delas empresas exportadoras únicamente tenemos información de 1993 y 2000, procedente de la misma 
fuente, pero en estecaso, dichainformación provienedeuna encuesta y, por lo tanto, estos últimos datos son una muestra correspondiente a una población desconocida. Así pues, no coinciden los datos anuales de exportación e importación con la información correspondiente a las características de las empresas dado que no están revisadas todas el mismo año, el organismo pertinente no guarda los datos para formar series históricas, y las empresas, aunque citan el total exportado y los países de destino, no especifican el montante correspondiente a cada país.

El análisis del flujo de las importaciones nos ha permitido deducir (ver cuadro 1) que: a) La estructura de las importaciones AndalucíaMarruecos (1989-2000) no ha cambiado sensiblemente a lo largo del periodo analizado, centrándose, según nuestros datos, en cuatro grandes grupos: productos alimentarios (74\% productos del mar), productos de origen mineral ( $82 \%$ materiales construcción), semiproductos (abonos, productos siderúrgicos y químicos) y productos finales (textil y confección, manufactura del cuero). Si bien los sectores importadores más importantes tienden a presentar una disminución en su conjunto pasando de $95.85 \%$ en $1989-1992$ a $79.25 \%$ en 1997-2000; existen dos sectores que muestran el cambio de tendencias que se está produciendo en la economía andaluza hacia la internacionalización. Así, podemos ver que los productos agroalimentarios entre 1989 y 2000 comienzan a tener cierto peso en el rubro de las importaciones (ver cuadro 1), sobre todo los transformados. De la misma manera, cabe destacar la relevancia que están cobrando los artículos de confección y punto.

b) Si comparamos los resultados de las entrevistas efectuadas a organismos de comercio exterior españoles con el análisis del cuadro 1 deducimos que ciertas empresas andaluzas importan porque: 1) una parte del proceso productivo (ingeniería, construcción, energía, pesca) lo subcontratan a firmas de Marruecos; 2) otras contratan algunas veces la compra de la producción (agricultura, alimentación, energía, pesca) a empresas de ese país. En este sentido, sabemos que las firmas Rhône-Alpes, Cataluña, Piamonte y Portugal tienen como clientes las empresas marroquíes de plásticos, electricidad y metal. Sin embargo, de acuerdo con la información recogida en los 
CUADRO 1 Valor delosproductosimportadosdeMarnuecos(en porcentaje).

\begin{tabular}{|l|r|r|r|}
\hline & $1989-1992$ & $1993-1996$ & $1997-2000$ \\
\hline Pescados & 35.74 & 33.06 & 31.56 \\
Sal, azufre, yeso & 31.71 & 31.19 & 27.61 \\
Minerales, escorias & 10.78 & 11.76 & 6.42 \\
y cenizas & & & \\
Confección y punto & 2.36 & 8.30 & 10.01 \\
Productos químicos & 3.08 & 3.98 & 2.35 \\
Manufactura de cuero & 10.85 & 1.13 & 0.35 \\
Fundición de hierro & 0.71 & 0.60 & 0.86 \\
y acero & & & \\
Productos agrarios & 0.62 & 1.17 & 2.88 \\
sin manipulación & & & 8.30 \\
Productos agrarios & 1.10 & 3.49 & \\
transformados & & & \\
\hline
\end{tabular}

FUENTE: Elaboración propia a partir de datos del reporte "Comercialización productos andaluces", Junta deA ndalucía, 2000.

organismos de comercio exterior, Andalucía todavía no está interesada por este tipo de negocios, salvo en los casos de las industrias textil, de confección, agroindustria, energía e ingeniería aplicada (las dos primeras ramas industriales contratan empresas del país alauita y también invierten allí, pero siempre el patronaje y al gunas veces el tejido procede de España, mientras que la agroindustria andaluza exporta desde Andalucía frutas y hortalizas para procesarlas en Marruecos); 3) otras firmas andaluzas importan porque han invertido en Marruecos para abaratar costes, localizando en ese país una parte del proceso productivo (transportes, alimentación, agrícolas, metal, textil y confección, químicas y plásticos, madera y mueble) mediante la creación de empresas mixtas, sucursales y el desarrollo de la subcontratación (A bengoa).

c) De acuerdo con el cuadro 1 habría que diferenciar entre 1) aquellos productos importados en los cuales Andalucía es productora y rival (confección y punto, manufactura del cuero, productos agrarios sin manipular y productos agrarios transformados); 2) las producciones que constituyen inputs para la industria andaluza (azufre y 
cemento; y minerales, escorias y cenizas); y 3) aquellos otros que se necesitan para satisfacer la demanda de la población andaluza (por ejemplo, los productos pesqueros). Desde ese punto de vista, cabría decir que Andalucía está pasando de ser un rival de Marruecos en las producciones agrarias y turísticas, existiendo todavía muchas suspicacias por parte del pueblo andaluz (organizaciones agrarias, pesqueras, empresarios, etcétera), para convertirse lentamente en inversor y transferidor de tecnología al país alauita, como veremos a continuación.

\section{FLUJO DE LAS EXPORTACIONES}

Por otra parte, si observamos el flujo de las exportaciones AndalucíaMarruecos, la principal característica es la gran diversificación de las mismas, dominando los productos manufacturados, los cuales representan $95 \%$ del total. Así, destacan los combustibles y aceites minerales, los textiles y la confección, y los productos siderúrgicos y sus manufacturas (ver cuadro 2). Cuando agrupamos a las empresas por productos exportados, vemos que las exportaciones se concentran en torno a los sectores agrario y maquinaria (ver cuadro 3), pero que entre 1993 y 2000 disminuye esa polarización (81\% en 1993, frente a un $65.87 \%$ en 2000), y tiende a una mayor diversificación. No obstante, este resultado es conveniente contrastarlo con otros datos para ver si la muestra de empresas está sesgada. En este caso, lo cotejamos con la Serieanual de comercio exterior que muestra el flujo deexportación (expresado en millones de pesetas), cuyos datos los podemos ver en el cuadro 2, y observamos que los índices de exportación no sólo son crecientes, sino que además, los sectores que destacan en los dos tipos de datos son los mismos, a excepción de los del papel, los plásticos, y los combustibles y aceites minerales, lo cual quiere decir que las ramas que no están representados en el cuadro 3 son aquéllas que están constituidas por grandes empresas, y en Andalucía únicamente hay establecidas dos o tres plantas de cada una de ellas, por lo que, es probable que no estuvieran incluidas en la muestra.

A hora bien, teniendo en cuenta queel mercado marroquí es emergente, una de las cuestiones más importante a destacar es que dentro 
de cada sector los flujos de exportación son inestables. Por eso, nos hemos visto obligados a tratar la serie con medias móviles, por tanto, parece claro que dentro de cada rama al gunas de las exportaciones son esporádicas y persisten fuertes dientes de sierra desequilibradores de los flujos; ejemplo de ello es el caso de los productos químicos y del material de transporte (aéreo y marítimo) dentro del grupo de maquinaria y bienes de equipo (ver cuadro 2). Por último, es conveniente resaltar específicamente la estabilidad del flujo exportador de maquinaria y bienes de equipo (aunque no se pueda observar en el cuadro 2; así como el creciente aumento de los textiles y la confección, que se ha convertido en un grupo fundamental de la exportación andaluza a Marruecos.

En este punto parece interesante estudiar las pautas de comportamiento de las exportaciones andaluzas según se dirijan a la UE o a países emergentes como Marruecos. Para ello, analizamos las empresas

CUADRO 2. Valor delosproductosexportadosaMarruecos (en porcentaje).

\begin{tabular}{|c|c|c|c|}
\hline & 1989-1992 & 1993-1996 & $1997-2000$ \\
\hline $\begin{array}{l}\text { Combustibles } \\
\text { y aceites }\end{array}$ & 9.05 & 26.70 & 28.35 \\
\hline Textil y confección & 11.57 & 21.33 & 20.97 \\
\hline $\begin{array}{l}\text { Maquinaria, bienes, } \\
\text { equipo y material } \\
\text { de transporte }\end{array}$ & 47.96 & 11.99 & 12.43 \\
\hline $\begin{array}{l}\text { Fundición de hierro, } \\
\text { acero, cobre y } \\
\text { manufacturas }\end{array}$ & 9.43 & 9.63 & 9.28 \\
\hline $\begin{array}{l}\text { Productos químicos } \\
\text { (orgánicos e inorgánicos) }\end{array}$ & 5.04 & 10.48 & 4.38 \\
\hline Papel y manufactura & 2.33 & 4.20 & 2.77 \\
\hline $\begin{array}{l}\text { Flor, semillas y grasas, } \\
\text { agrarias }\end{array}$ & 1.32 & 2.54 & 4.07 \\
\hline Materiales plásticos & 0.53 & 2.15 & 2.23 \\
\hline
\end{tabular}

FUENTE: Elaboración propia a partir de datos del reporte "Comercialización productos andaluces". 
CUADRO 3. Sectores más importantes exportados 1993, 2000.

\begin{tabular}{|l|c|c|}
\hline \multicolumn{1}{|c|}{$\begin{array}{c}\text { Sectores más importantes } \\
\text { exportados }\end{array}$} & 1993 (en \%) & 2000 (en \%) \\
\hline Agrario & 16.00 & 13.92 \\
Semillas & 8.00 & 2.00 \\
Maquinaria, bienes y & 26.00 & 19.00 \\
equipamiento & & \\
Textil y confección & 4.00 & 6.96 \\
Derivados metálicos & 7.00 & 6.09 \\
Servicios agrícolas & - & 4.34 \\
Siderurgia & 4.00 & 3.48 \\
Productos químicos & 7.00 & 3.48 \\
Productos alimenticios & 5.00 & 5.20 \\
Material de transporte & 8.00 & 1.40 \\
\hline
\end{tabular}

FUENTE: Elaboración propia a partir de datos del reporte “Comercializadora de productos Andaluces", Junta deA ndalucía.

que exportan a A lemania y a M arruecos según las variables deempleo, volumen deventasy porcentajeexportado. Deacuerdo con estos rasgos, no existen diferencias notables entre las empresas que exportan a ambos países, en tanto que el nivel de ventas parece más alto en aquéllas que lo hacen a Alemania (ver cuadro 4).

A decir verdad, la diferencia de mayor importancia radica en el rubro de productos exportados, donde $50.12 \%$ de las firmas que lo exportan a Alemania son empresas agrarias (productos agrícolas y primera transformación, como aceite, frutos secos y sal). Si a ese porcentaje le sumamos los productos artesanales (3.22\%), tenemos $53.04 \%$, y que junto al de productos agroindustriales, alcanza la cifra de $67.73 \%$ (ver cuadro 5). Sin embargo, las empresas que exportan a Marruecos son menos de la mitad que las que exportan a Alemania (con base en la muestra), pero los productos exportados se caracterizan por ser fundamental mente manufacturados y servicios: maquinaria y bienes equipo, $20 \%$; textil y confección, $6,96 \%$; servicios a agricultura, 4.34\%; siderurgia, 3.48\%; productos derivados 
CUADRO 4. Volumen deventas delasempresasexportadorasaMarruecos y Alemania, 2000 (en millones depesetas).

\begin{tabular}{|l|r|r|}
\hline Ventas & \% Empresas de Alemania & \%Empresas de Marruecos \\
\hline $1<25$ & 4.69 & 5.00 \\
$25<125$ & 17.30 & 19.17 \\
$125<625$ & 33.43 & 30.83 \\
$625<312531$. & 67 & 31.67 \\
$3125<15625$ & 10.56 & 10.00 \\
$15625<78125$ & 1,17 & 2,50 \\
$78125<390625$ & 0.88 & 0.83 \\
$390625<1953125$ & 0,29 & - \\
TOTA L & 100.00 & 100.00 \\
\hline
\end{tabular}

FUENTE: Elaboración propia a partir del reporte “Comercializadora productosAndaluces", Junta deAndalucía.

del metal, 6.09\%; productos químicos, 3.48\%, y productos agrícolas $13.91 \%$ (flor cortada, hortal izas, etcétera).

Así pues, Andalucía, en el rubro de los productos manufacturados, se comporta como un país de tecnología media, demostrando que está especializado en la exportación de maquinaria agrícola e industrial, material de transporte, maquinaria de oficina y material eléctrico y electrónico, así como metálicas básicas, alimentación, bebidas y tabaco, madera y otras industrias manufactureras. Claro está que el grueso de las exportaciones andaluzas se dirigen a la UE (64.26\% de las ventas internacionales de la región), mientras que la exportación a Marruecos tan sólo representa $1 \%$ del total. A demás, de ese $64.26 \%$ que se dirigea la UE, $40 \%$ correspondeal sector agrario más los productos agroindustriales (Moral Pajares, 1998).

PARTICIPACIÓN ANDALUZA EN EQUIPAMIENTOS E INFRAESTRUCTURAS

Por último, queda por analizar la capacidad de A ndalucía para participar en la construcción de infraestructura y equipamiento 
CUADRO5.Distribución deempresasexportadorasaA lemaniapor sectores, 1998.

\begin{tabular}{|l|r|}
\hline \multicolumn{1}{|c|}{ Sectores } & \multicolumn{1}{|c|}{$\%$} \\
\hline Productos agrarios & 44.85 \\
Primera transformación de productos agrarios & 5.27 \\
Segunda transformación de productos agrarios & 14,37 \\
Productos artesanales & 3,22 \\
TOTAL & 67,73 \\
\hline
\end{tabular}

FUENTE: Elaboración propia a partir de datos del reporte "Comercializadora de productos A ndaluces", Junta deA ndal ucía.

(puertos, centrales eléctricas, térmicas, aeropuertos, telecomunicaciones, sanidad, equipamiento para colectividades locales, instalaciones para la transformación agraria, minería [equipos de extracción], electrificación, etcétera) en Marruecos.

Para estudiar este punto entrevistamos a cuatro Consejerías de la Junta de Andalucía, y a cinco grandes empresas andaluzas que trabajan el mercado marroquí, para ver qué proyectos estaban desarrollando en esa población, puesto que se trata de un ámbito en el que intervienen grandes y medianas empresas.

Evidentemente, en este apartado entramos de lleno en la llamada cooperación institucional efectuada por Andalucía a través de las ayudas establecidas por la UE, el Estado Español y la Junta de Anda-

CUADRO 6. EmpresasexportadorasaMarruecossegún \%exportado, 2000.

\begin{tabular}{|l|r|}
\hline $0<5$ & 25 \\
$5<10$ & 19.17 \\
$10<20$ & 19.17 \\
$20<40$ & 15.00 \\
$40<80$ & 16.67 \\
$80<100$ & 5.00 \\
\hline
\end{tabular}

FUENTE: Elaboración propia a partir del reporte “Comercializadora de productosAndaluces", Junta deA ndal ucía. 
lucía, (cabe mencionar que buena parte de estas subvenciones se transfieren al sector privado andaluz a través de contratos de obras o suministros de bienes y servicios). A simismo, hay que considerar que las actuaciones evaluadas en este apartado se concentran en las regiones marroquíes de Tánger-Tetuán, Taza-Al Hoceima y Oriental.

Hasta ahora, la cooperación andaluza en Marruecos sobre infraestructura y equipamiento, se ha centrado en cuatro ámbitos: sanidad, energía, recursos hidráulicos y equipamientos urbanos. En el campo energético es donde mayores resultados se han conseguido, al intervenir las empresas andaluzas en el desarrollo conjunto de infraestructuras de transporte energético y en la construcción de una central térmica dedicada a la exportación de energía eléctrica.

Desde la administración andaluza-marroquí, estas acciones se han interrelacionado con programas encaminados al desarrollo de equipamientos locales y regionales de electrificación (energía solar y fotovoltaica), con proyectos de racional ización de energía, de transferencia de tecnología fotovoltaica y con el proyecto denominado Prosol, a fin de conseguir un mayor al cance de los impactos. Por ejemplo, este último proyecto seenmarca dentro de un programa nacional marroquí de difusión de sistemas solares térmi cos a gran escala, que tiene como objetivo la intensificación de la utilización colectiva e individual de los colectores solares. Además, se ejecutan diversas acciones complementarias; como lo serían la formación de técnicos y la realización de auditorías en instalaciones industriales, además de que abarca los sectores agroalimentario, textil, cerámica, salud, consumo doméstico, turismo y agrícola. Entre 1994 y 2000, el gasto materializado total por las empresas ejecutoras de las obras se cifra en 347000000 de pesetas pagados, equivalentes a 1862000 de dólares.

En materia de salud, las inversiones se han centrado en la construcción de equipamientos de atención primaria y en la mejora y modernización dehospital es (área de Tetuán y Xauen), con un presupuesto ejecutado y materializado de 326000000 de pesetas entre 1994 y 2000 (equivalentes a 1749000 de dólares). La realización de estas obras ha estado acompañada de programas dedicados a la formación y reciclaje del personal sanitario, de vigilancia epidemiológica y de la mejora de medios técnicos y de diagnóstico. 
Dentro de la planificación hidráulica cabe destacar un programa de ordenación, recuperación y mejora de distintas cuencas, entre ellas la de Oued-Laou (programa hasta ahora ejecutado en un $50 \%$ ), el desarrollo de otro para el abastecimiento de agua potable en las provincias de Chefchauen y Tetuán, y la construcción de un centro de transferencia tecnológica en materia de aguas (Junta de Andalucía, 2000). En total, las empresas andaluzas intervinientes llevan ejecutad os cerca de 142000000 de pesetas entre 1994 y 1999 (equivalentes a 762000 de dólares). Además, estas acciones están siendo complementadas con otras dedicadas a la transferencia tecnológica sobre estos temas.

Por último, en el área de arquitectura, la cooperación andaluza se plasma en la rehabilitación de centros históricos y en la construcción de equipamientos urbanos con el fin de conseguir resultados positivos en el desarrollo turístico y cultural de las poblaciones localizadas en el norte de Marruecos (Taza, Taounate, Chefchaouen). Hasta ahora, tan sólo se ha efectuado $38 \%$ de los pagos comprometidos de una inversión total de 204000000 de pesetas entre 1994 y 2000 (equivalentes a 1094000 de dólares).

Dada la escasa tradición de la cooperación andaluza-marroquí, las iniciativas desarrolladas en materia de infraestructura y equipamiento no alcanzan todavía el nivel deseado debido a la falta de experiencia de las empresas y de las instituciones andaluzas, lo que ha dado como resultado un bajo alcance de los compromisos entre ambas partes.

Por otro lado, tenemos que reconocer que todavía no ha transcurrido el tiempo suficiente para poder evaluar -ex post- con detalle los logros alcanzados en los distintos programas aplicados. A un así, como la evaluación (intermedia) de los impactos podría venir definida por el efecto acumulativo sobre el territorio de una serie de programas complementarios, hemos investigado el nivel de sinergia de unos proyectos con otros, dando un índice de 7 sobre 10. Desde el punto de vista de la gestión financiera hemos comprobado hasta qué punto se han verificado las previsiones de gastos realizados. En este sentido, cabe afirmar que el porcentaje de pagos materializados sobre el gasto elegible ha sido bastante alto entre 1994 y 2000, tal como queda demostrado. 


\section{CONCLUSIONES}

Tradicionalmente, Andalucía y Marruecos han sido rivales en el terreno económico, incluso ahora existen reticencias por parte de las organizaciones agrarias, pesqueras, etcétera, que no entienden bien la política de la UE ni de la Administración Central Española.

El desarrollo de la globalización, del mercado único y la política euromediterránea están forzando a Andalucía a generar inversiones directas y a incrementar las relaciones comerciales y de cooperación institucional con Marruecos. Evidentemente, ha transcurrido muy poco tiempo desdequesecré la zona delibrecomercio UE-Marruecos (1996) para poder detectar algún resultado importante, pero si comparamos los productos importados y exportados a Marruecos, valorados en millones de pesetas, encontramos que:

A) Hay sectores donde existe un gran paralelismo en cuanto a la magnitud del flujo de entrada y salida, lo cual quiere decir que esos subsectores importadores y exportadores son complementarios, por ejemplo, el de productos químicos orgánicos e inorgánicos, y el de sal , azufre, cemento y yeso. Cuando, además, coinciden las empresas que exportan e importan, significa que éstas han hecho inversiones directas en Marruecos, como sería el caso de los rubros de grasas, aceites animales y vegetales, el de fundiciones de hierro y acero y sus manufacturas, y el de textil y confección. En estos casos, la economía andaluza, tanto en esos productos como en esos tipos de relaciones, cabe decir está internacionalizada al objeto de 1) abaratar costes y reexportar a otros países, aumentando la cuota de mercado; 2) para abaratar costes y vender a los mismos mercados que antes; y 3) para abaratar costes e introducirse en el mercado marroquí a fin de construir infraestructuras y equipamientos, comercializar sus productos, etcétera. Sin embargo, de acuerdo con la información que tenemos, conviene llamar la atención que no son tantas las empresas andaluzas que han desarrollado ese tipo de relaciones complejas con Marruecos.

B) Hay sectores donde no existe paral elismo entre los flujos de las importaciones y las exportaciones valoradas en millones de pesetas. Esto ocurre en los rubros de semillas y plantas; extractos, curtientes, 
materias colorantes y barnices; materias plásticas y sus manufacturas; papel, cartón y sus manufacturas; maquinaria y bienes de equipo; material de transporte; aparatos e instrumentos de óptica; mobiliario quirúrgico; y el de energía solar y fotovoltaica, lo cual quiere decir que con estos productos Andalucía se comporta como un área desarrollada de tecnología media, tiene ventajas sobre estos productos, y las empresas que los exportan no sólo los comercializan en Marruecos, sino también en países desarrollados. Además, a través de estos productos Andalucía está exportando tecnología incorporada y está favoreciendo la modernización del tejido industrial marroquí.

Por lo tanto, hasta ahora, Andalucía está cumpliendo su papel de región fronteriza al contribuir al desarrollo de un espacio transfronterizo, sobre todo en la exportación de productos manufacturados y mediante la realización de inversiones empresariales en $\mathrm{Ma}$ rruecos. Pero falta sensibilizar más al tejido de PYMES andaluz acerca del interés derealizar inversiones en Marruecos y decolaborar con ellos más estrechamente, puesto que existe todavía un distanciamiento importanteentrelos tejidos empresariales y agentes económicos marroquíes y andaluces.

Por otra parte, se requeriría quelas grandes empresas establecidas en Andalucía intervinieran más activamente en la construcción de infraestructuras y equipamientos. Precisamente los sectores de construcción de instalaciones para la transformación de tierras en regadío, instalaciones energéticas, equipamientos para colectividades locales y hospitalarias, junto con la maquinaria, son los sectores que mejores perspectivas de futuro tienen en la exportación y en las inversiones directas a Marruecos. 


\section{BIBLIOGRAFÍA}

Moral Pajares, E. 1998. A juste del comercio exterior andaluz tras la adhesión de España a la U E, Baeza (Jaén), Universidad de Jaén y Caja Sur.

Comercializadora de Productos Andaluces. 1994. Guía de exportadores de A ndalucía. Sevilla, IFA, Junta de Andalucía.

Junta de Andalucía. 2000. A ctuaciones desarrolladas por la Junta de A ndalucía en el marco dela iniciativa comunitaria Interreg II, E spañaM arruecos (1994-1999). Málaga, Junta de Andalucía, Universidad de Málaga.

\section{OTRAS FUENTES}

Entrevistas realizadas en abril-septiembre de 1998: Confederación deEmpresariosAndaluces (CEA); Departamento dePromoción Exterior del Instituto de Fomento Andaluz; Oficina Comercial de España en Rabat; Comercialización de Productos AndaIuces; Dirección General de Relaciones con la UE; Cámara de Comercio de Cádiz; Consejería de Agricultura, Medio A mbiente, Obras Públicas; y a 11 empresas (A bengoa, Siemens, Sevillana Electricidad, Ubago, etcétera). 
